- The removal of mild to moderately impacted wisdom teeth can be consistently and reliably performed in the 'high street' practice situation.

- Complication rates can be within those standards currently published.

- As with all day surgery, the potential availability of a 'hospital bed' if required, should be a consideration.

\title{
Lingual nerve injury subsequent to wisdom teeth removal - a 5-year retrospective audit from a high street dental practice
}

\author{
N. J. Malden ${ }^{1}$ and Y. G. Maidment ${ }^{2}$
}

Lingual nerve damage subsequent to lower wisdom tooth removal affects a small number of patients, sometimes producing permanent sensory loss or impairment. A number of surgical techniques have been described which are associated with low incidences of this distressing post-operative complication. When a technique is adopted by an individual clinician then a personal audit may be prudent to establish how effective it is in relation to established nerve injury rates. This audit looks at a technique involving the minimal interference of lingual soft tissues during lower wisdom tooth removal in a high street practice situation for patients having mild to moderate impacted wisdom teeth removed under local anaesthetic. It was concluded that the technique employed was associated with a low incidence of lingual nerve trauma, comparable with that reported elsewhere.

McGurk and Haskell attempted recently to rationalise the arguments surrounding the relationship of surgical technique and of operator to lingual nerve morbidity during wisdom tooth removal. In considering techniques involving the raising of a lingual soft tissue flap off the lingual plate in comparison with those techniques where a lingual flap is not raised beyond that required to visualise the crest of the lingual plate, the following statement was made: 'Specialists with an optimal skill base should be able to use either technique as long as audit shows that their performance is better than currently published standards. ${ }^{1}$

This audit considers a technique which has been adopted by the author (NJM) as that most appropriate in his hands, for the removal of mild to moderately impacted

${ }^{1 *}$ Associate Specialist, Oral Surgery Department, Edinburgh Dental Institute, Lauriston Building, Edinburgh EH3 9YW;

2Practice Principal, 2 Chester Street, Edinburgh EH3 7RA

${ }^{*}$ Correspondence to: Nick Malden

E-mail: Nick.Malden@Ipct.scot.nhs.uk

\section{Refereed Paper}

Received 06.12.01; Accepted 18.04.02

$\odot$ British Dental Journal 2002; 193: 203-205 wisdom teeth for adults treated as outpatients in general practice using local anaesthesia.

\section{NOTE ON TERMINOLOGY}

Classification of angulation and depth of tooth are radiographically assessed by applying Winter's lines or certainly the principles behind them. The authors accept that Winter originally described the technique for use with a periapical film $^{2}$ but would suggest that the principles are the same when applying them to an OPG.

When further assessing an impaction the author (NJM) would be taking into account the patient's age, previous experience and tolerance of treatment with local anaesthesia, presence of a "valuable' restoration in an adjacent molar, degree of access and presence of a disruptive gagging reflex. Further radiographic signs of an intimate relationship of the tooth to the inferior dental canal would also be noted. ${ }^{3}$

The author would generally be placing those teeth estimated to be requiring $>10$ minutes operating time to remove as mild impactions, while those requiring between 10-20 minutes operating time as moderate. Perhaps the terms 'mild to moderate difficulty' could better replace the terms 'mild to moderate impaction'. Included in the audit are also patients who received single agent (Midazolam) intravenous conscious sedation. The technique adopted has been described in the literature. ${ }^{4,5}$ It is of note that one recent publication describes a technique with an apparently very low incidence of permanent nerve damage. ${ }^{6}$ The technique employed was the lingual split technique, with patients under general anaesthesia. The authors would consider that to compare nerve injury rates with a technique which is only performed under general anaesthesia (the lingual split technique) is not appropriate when considering the management of impacted wisdom teeth in a 'high street' practice, if for no other reason than general anaesthesia not being available in dental practices.

The audit project was primarily to look at the incidence of derangement of tongue sensation subsequent to surgical removal of lower wisdom teeth by a single operator (NJM). 


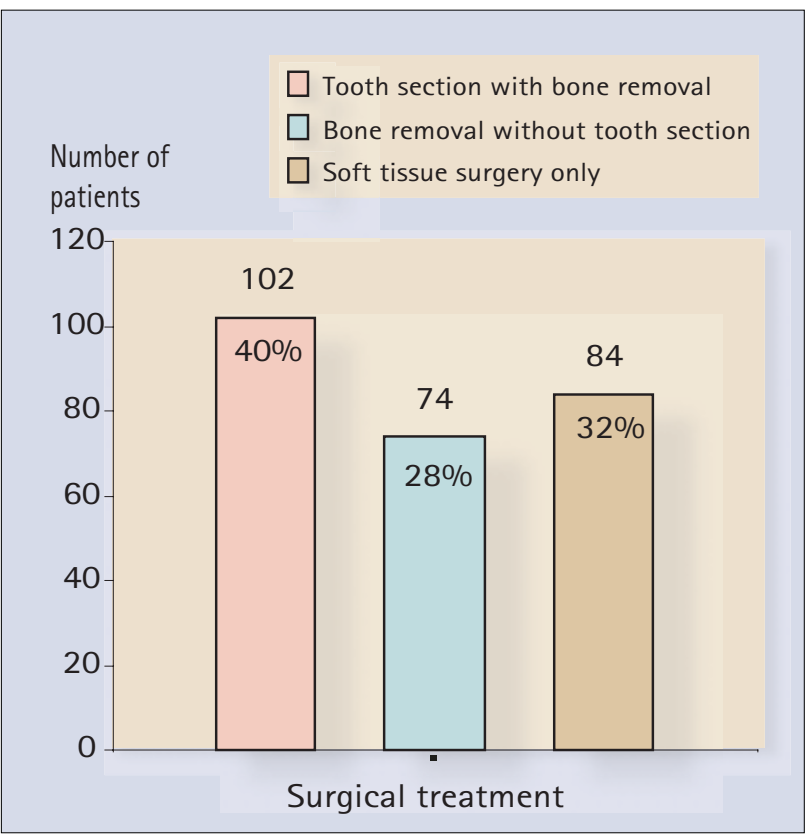

Fig. 1 Form of surgical treatment employed

\section{AIMS}

- To determine if the incidence of lingual nerve damage differed to any significant extent from that reported elsewhere.

- To modify the operative technique, if indicated, to bring the incidence of nerve damage to within an accepted currently published standard.

It may be noted that although the incidence of a number of complications of wisdom tooth removal have been shown to vary considerably with different techniques and differing authors the incidence of nerve damage (inferior alveolar and lingual, branches of the trigeminal) are considered significant morbidity indicators. Two reasons account for this:

- Damage to these nerves can lead to loss of sensation of tongue and lower lip which if permanent can be considered a disability of some severity,

- Some authors consider this nerve damage to be avoidable and directly attributable to the operative technique employed.

Robinson and Smith have stated that 'the permanent lingual nerve injury often results from direct damage from a rotating bur. ${ }^{4}$ In a more recent paper, Robinson, Loescher and Smith found that 'The affected nerves were always found trapped in scar tissue and sometimes expanded to form a 'neuroma'. In about 50\% complete division of the nerves was evident, small fragments of metal were sometimes found embedded within the epineurium and scar tissue, presumably having been shaved from the lingual retractor (Howarths elevator) during the initial operation: ${ }^{7}$ following technique was employed. The tooth was incompletely sectioned with a bur and then a Coupland's chisel placed into the cut and rotated to complete the division. The removal of tooth crowns would often be performed early to allow continuation of bone removal distally under direct vision. The 'time honoured' rule of loosening any tooth before sectioning it, being adhered to if possible. Following elevation of tooth and roots, sutures were placed following the rule of 'tight knots not sutures', this being to deal with the consideration that should an unusually placed lingual nerve be caught by the suture that it was not 'strangled'. In the cadaver study by Kiesselbach $\&$ Chamberlain, 17\% of cases demonstrated a lingual nerve at or above the crest of the lingual plate. One case is described where the nerve passed through the retromolar pad, $2 \mathrm{~mm}$ above the crest of the lingual plate. $^{8}$ Also Pogrel et al. in a cadaver study demonstrated in 15\% of cases a lingual nerve level with, or superior to, the crest of the lingual plate. ${ }^{9}$

\section{EMPLOYED}

All procedures were carried out by the first author (NJM). In all cases a mucoperiosteal flap was raised buccal to the lower third molar. The distal relieving incision was placed lateral to the retromolar pad in such a position that all anatomical variants of the lingual nerve position would be avoided., ${ }^{8,9}$ It has generally been accepted that an incision following the external oblique ridge will be safe in this respect. The buccal flap was retracted with a Bowdler Henry rake retractor or Austin retractor. Lingual tissue was retracted only to expose the occlusal aspect of the tooth or the superior aspect of mandibular bone covering the tooth or the crest of the lingual plate. No attempt was made to raise or elevate a lingual mucoperiosteal flap off the lingual aspect of the mandible. A Howarth retractor was placed to retract only the lingual 'cuff' of soft tissue so allowing direct vision of the bur cutting tip if distal bone was removed, care being taken to cut from the lingual towards the buccal. If tooth section was performed then to avoid perforation of the lingual plate the

\section{STUDY METHOD}

A pilot survey of 25 patient's notes was carried out at one location to test the feasibility of information gathering, using a data collection table. Following this 260 lower wisdom tooth removals were identified by retrospectively examining patient's records from four separate practice locations. The patients involved in the study were taken from the first author's own daybook and represented consecutively treated wisdom teeth patients between 4/12/89 and 13/09/95 (a period of almost 6 years).

\section{RESULTS}

All 260 extractions were surgical. In all cases a buccal soft tissue flap was raised. $102(40 \%)$ involved sectioning of tooth with bone removal. Seventy-four (28\%) involved bone removal only (Fig. 1).

Complications reported were (Table 1):

- Swelling 15 (5.7\%)

- Bleeding 3 (1.1\%) (one patient was admitted to the local maxillo-facial unit to facilitate management of

\begin{tabular}{lrrl} 
Table 1 Complications reported & & & \\
\hline Complication & No & $\%$ & Comment \\
\hline Swelling & 15 & $5.7 \%$ & \\
Bleeding & 3 & $1.2 \%$ & $\begin{array}{l}\text { One patient admitted to local } \\
\text { maxillo-facial unit overnight }\end{array}$ \\
Post-operative pain & 5 & $1.9 \%$ & \\
Infected sockets & 13 & $5.0 \%$ & \\
Trismus & 2 & $0.8 \%$ & Return of full sensation within 6 weeks \\
Lingual paraesthesia & 1 & $0.4 \%$ &
\end{tabular}


postoperative haemorrhage)

- Post-operative pain 5 (1.9\%)

- Infected socket 13 (5\%)

- Trismus $2(0.8 \%)$

- Lingual Paraesthesia 1 (0.4\%)

(full return of normal sensation within 6 weeks)

Other entries within notes included:

- Bone wax placed $1(0.4 \%)$

- Antibiotics given 31 (11.8\%)

\section{DISCUSSION}

While gathering information on lingual nerve damage the opportunity was taken to look for other post extraction complications. As regards the temporary nerve damage incidence of $0.4 \%$, this figure compares very favourably with that reported by $\mathrm{H}$ McGurk R Haskell ${ }^{1}$ for bone removal withdrill no lingual flap (UK) where the temporary nerve injury was reported as $0.8 \%$ and permanent injury reported as $0.3 \%$.

The only technique recording a lower incidence of permanent lingual nerve injury in the UK was the lingual split with lingual flap retractor technique which is normally performed under intubation general anaesthesia. 6
A criticism of our study might include its retrospective nature, the relatively small number of teeth requiring bone removal with sectioning of tooth compared to expected incidence of complication and that this also implies shallower impactions than one might expect to be treated in a maxillo-facial unit. It is accepted by the authors that many of the post operative complaints listed may well go underreported by patients but the authors suggest that post-operative sensory nerve damage is not a complication that patients fail to report very often.

\section{CONCLUSION}

The surgical technique described is associated with an acceptable low incidence of lingual nerve damage when considering its application in a general practice situation for mild to moderately impacted lower third molars. The recorded incidence of post-operative pain (1.5\%) and infected sockets $(5 \%)$ are areas where a continued prospective audit may be beneficial.

The author would like to thank Mr Terry Simpson, Dental Audit Facilitator of Lothian Health Board for his input and support for this project. Also thanks to John Stevenson and Kenny Combes for help in providing information on their patients.

1 McGurk M, Haskell R. Wisdom tooth removal and lingual nerve damage. Br J Oral Maxillofac Surg 1998; 37: 253-254.

2 Howe G L. Minor Oral Surgery. 2nd ed, pp93 - 103. Bristol: John Wright \& Sons Ltd, 1971.

3 Rood J P, Nooraldeen Shehab B A A. The radiological prediction of inferior alveolar nerve injury during third molar surgery. Br J Oral Maxillofac Surg 1990; 28: $20-25$.

4 Robinson P P, Smith K G. Lingual nerve damage during lower 3rd molar removal: a comparison of two surgical methods. Br Dent J 1996; 180: 456-461.

5 Appiah-Anane S, Appiah-Anane M G. Protection of lingual nerve during operations on the mandibular third molar: a simple method. Br J Oral Maxillofac Surg 1997; 35: 170-172.

6 Moss CE, Wake J G. Lingual access for 3rd molar surgery: a 20 year retrospective audit. Br J Oral Maxillofac Surg 1999; 37: 255-258.

7 Robinson P P, Loescher A R, Smith K G. A prospective, quantitative study on the clinical outcome of lingual nerve repair. Br J Oral Maxillofac Surg 2000; 38: 255263.

8 Kiesselbach J E, Chamberlain J G. Clinical and anatomic observations on the relationship of the lingual nerve to the mandibular third molar region. J Oral Maxillofac Surg 1984; 42: 565-567.

9 Pogrel M A, Renaut A, Schmidt B, Ammar A. The relationship of the lingual nerve to the mandibular third molar region. J Oral Maxillofac Surg 1995; 53: 1178-1181. 